\title{
Per-antenna hardware optimization and mixed resolution ADCs in uplink massive MIMO
}

Daniel Verenzuela, Emil Björnson and Michail Matthaiou

\section{Conference article}

Cite this conference article as:

Verenzuela, D., Björnson, E., Matthaiou, M. Per-antenna hardware optimization and mixed resolution ADCs in uplink massive MIMO, In Michael B. Matthews (ed.) Conference Record of The Fifty-FirstAsilomar Conference on Signals, Systems \& Computers, IEEE conference proceedings; 2017, pp. 27-31. ISBN: 978-1-5386-1823-3

Signals, Systems \& Computers eISSN: 2576-2303, 2017

DOI: https:// doi.org/ 10.1109/ACSSC.2017.8335129

Copyright: IEEE conference proceedings

The self-archived postprint version of this conference article is available at Linköping University Institutional Repository (DiVA):

http:// urn.kb.se/ resolve?urn=urn:nbn:se:liu:diva- 148777 


\title{
Per-Antenna Hardware Optimization and Mixed Resolution ADCs in Uplink Massive MIMO
}

\author{
Daniel Verenzuela*, Emil Björnson*, and Michail Matthaiou ${ }^{\dagger}$ \\ *Department of Electrical Engineering (ISY), Linköping University, Linköping, Sweden. \\ $\dagger$ Institute of Electronics, Communications and Information Technology (ECIT), Queen’s University Belfast, Belfast, U.K.
}

\begin{abstract}
Massive multiple-input mutiple-output (MIMO) is a key technology for next generation wireless networks that deploys many antennas at the base stations (BSs). This requires lowcomplexity hardware at each antenna branch that, in turn, increases distortions. This work studies the selection of per-antenna hardware quality in terms of analog-to-digital converters (ADCs) resolution. A new achievable spectral efficiency (SE) expression is derived and majorization theory is used to analyze the order preserving properties of the $\mathrm{SE}$ and the power consumption with respect to the per-antenna ADC resolutions. That is, given a fixed sum of $A D C$ resolutions across the antenna array, is it preferable to use an equal-ADC over a mixed-ADC approach? The results show that having equal-resolution ADCs across the antenna array maximizes the SE and minimizes the power consumption.
\end{abstract}

\section{INTRODUCTION}

Massive multiple-input multiple-output (MIMO) increases the spectral efficiency (SE) and energy efficiency (EE) of wireless networks by spatially multiplexing several user equipments (UEs) using many antennas at the base stations (BSs) [1], [2]. To implement a Massive MIMO BS, the per-antenna circuit cost and power consumption need to be reduced significantly in comparison to current BSs. This calls for lowcomplexity hardware at each antenna's radio frequency (RF) chain which introduces additional distortion into the system. Fortunately, the large number of antennas is able to mitigate most of these effects and significant gains in SE and EE can still be obtained with massive MIMO [3]-[5].

An important source of distortion and power consumption in a Massive MIMO BS is the analog-to-digital converters (ADCs). By reducing the resolution of the ADCs, the power consumption, hardware complexity and front end traffic can be reduced. On the other hand, low-resolution ADCs cause higher distortions that reduce the SE. However, this undesired impact on the SE can be mitigated by increasing the number of BS antennas. Several works have studied the selection of $\mathrm{ADC}$ resolution to maximize performance in terms of bit error rates, SE, and power consumption [5]-[8]. However, these works assume that all antenna RF chains have the same ADC resolution. In [9], [10], the authors studied the case in which some RF chains have high-resolution ADCs while the rest have low resolution, in order to find a trade-off between hardware complexity and SE. In these cases, significant gains in SE have been reported compared to using only low-resolution ADCs.

This paper has received funding from ELLIIT and the Swedish Foundation for Strategic Research (SSF). The work of M. Matthaiou was supported in part by the EPSRC under grant EP/P000673/1.
However, the effect of channel estimation in multiuser MIMO detection has not been considered and it has been assumed that switching mechanisms at sampling rate are available to adapt the ADC resolution of each antenna dynamically. This is difficult to implement in practice. In [11], the authors consider the more practical scenario with mixed ADCs that remain static over time. The paper studies the optimal proportion of antennas with either high- or low-resolution ADCs to maximize the SE under a power constraint. Numerical results indicate that using many BS antennas with only one-bit ADCs is the best approach. In our paper, mixed-resolution ADCs are studied in a practical scenario where the ADC resolution of each antenna can be set individually but remains static over time. An achievable SE expression considering different hardware impairment levels for each RF chain is derived. Majorization theory is then used to study the order preserving properties of the SE and the power consumption with respect to how unequal are the ADC resolutions across the array. The results show that equal-resolution ADCs across the array jointly maximize the SE and minimize the power consumption.

\section{SySTEM MODEL}

Consider the uplink of a single cell with $M \mathrm{BS}$ antennas serving $K$ single-antenna UEs. The communication channel is modeled as block fading where the channel realizations are considered static and frequency flat during a time period $T_{c}$ and for a bandwidth $B_{c}$. This time and frequency block is referred to as the coherence block and consists of $\tau_{c}=T_{c} B_{c}$ complex symbols. The total system bandwidth $B_{\mathrm{W}}$ is such that $B_{\mathrm{W}} / B_{c}$ is an integer. The channel between all BS antennas and UE $k$ is modeled as $\mathbf{h}_{k} \sim \mathcal{C N}\left(\mathbf{0}, \beta_{k} \mathbf{I}_{M}\right)$ where $\beta_{k}$ accounts for the large-scale fading effects. The BS receiver is assumed to be affected by hardware impairments, which are modeled as independent additive distortions. The energy of the distortion at the $m^{t h}$ antenna element is proportional to $\epsilon_{m}$. When $\epsilon_{m}=0$ there is no distortion which corresponds to the case of perfect hardware. The received signal at the BS for an arbitrary symbol in the coherence block is

$$
\mathbf{y}=\sum_{i=1}^{K} \sqrt{\rho_{i}} \mathbf{h}_{i} s_{i}+\mathbf{n}+\mathbf{e}
$$

where $\rho_{i}$ and $s_{i}$ are the transmission energy per symbol and the transmitted symbol from UE $i$, respectively, such that $\mathbb{E}\left\{\left|s_{i}\right|^{2}\right\}=1(\mathbb{E}\{\cdot\}$ stands for the expected value). The 
thermal noise at the BS is $\mathbf{n} \sim \mathcal{C N}\left(\mathbf{0}, \sigma^{2} \mathbf{I}_{M}\right)$. The level of hardware impairments is determined by the diagonal matrix $\mathbf{D}_{\epsilon}=\operatorname{diag}\left(\epsilon_{1}^{2}, \ldots, \epsilon_{M}^{2}\right)$ and the distortion noise is given by

$$
\mathbf{e}=\mathbf{D}_{\epsilon}^{\frac{1}{2}}\left(\sum_{i=1}^{K} \rho_{i} \mathbf{D}_{\left|\mathbf{h}_{i}\right|^{2}}+\sigma^{2} \mathbf{I}_{M}\right)^{\frac{1}{2}} \mathbf{r}
$$

where $\mathbf{D}_{\left|\mathbf{h}_{i}\right|^{2}}=\operatorname{diag}\left(\left|h_{1 i}\right|^{2}, \ldots,\left|h_{M i}\right|^{2}\right) \forall i \in\{1, \ldots, K\}$ (the notation $h_{m i}$ indicates the $m^{\text {th }}$ element of $\mathbf{h}_{i}$ ). The randomness of the distortions is modeled by $\mathbf{r} \sim \mathcal{C N}\left(\mathbf{0}, \mathbf{I}_{M}\right)$.

This parametrized model is commonly used for evaluating the overall system performance of the network under residual hardware impairment effects [3]. In this article, ADCs are considered to be the main source of distortions and the parametrized model is adapted as in [5] to serve as a firstorder approximation of the quantization distortions. Thus, $\epsilon_{m}=\zeta_{m} 2^{-b_{m}}$, where $1<\zeta_{m}<2$ is a parameter related to the saturation level of the ADC in the $m^{\text {th }}$ BS antenna element and $b_{m}$ is the ADC resolution. Note that this model provides good insights into the impact of varying the ADC resolutions due to the exponential decrease in distortion energy with $b_{m}$. Moreover, in (2), the distortion noise depends on the instantaneous realizations of the channel which gives good insights into the effects introduced by imperfect channel state information (CSI). On the other hand, analyzing the quantization operation with an exact model can provide more accurate results, however, this is often intractable and provides little insights. To offer practical validation of the theoretical analysis, in Section VI, numerical results are presented to compare the achievable SE with the parametrized model and an exact quantization model. The results show that the achievable SE expression with the parametrized model is accurate for ADC resolutions of at least 3 bits.

\section{Channel Estimation}

To estimate the channels at the BS, $K$ out of $\tau_{c}$ symbols are used by the UEs for transmitting mutually orthogonal pilot sequences. These sequences are defined as $\phi_{i} \in \mathbb{C}^{K}$ $\forall i \in\{1, \ldots, K\}$ with elements having unit modulus $\left|\phi_{j i}\right|=1$ $j \in\{1, \ldots, K\}$. The orthogonality condition is given by $\phi_{k}^{H} \phi_{i}=K$ for $i=k$ and $\phi_{k}^{H} \phi_{i}=0$ for $i \neq k$. The received signal at the BS from all pilot symbols is

$$
\mathbf{Z}=\sum_{i=1}^{K} \sqrt{\varrho_{i}} \mathbf{h}_{i} \boldsymbol{\phi}_{i}^{T}+\overline{\mathbf{N}}+\mathbf{\Upsilon}
$$

where $\varrho_{i}$ is the transmission energy per pilot symbol from UE $i$. The thermal noise is given by $\bar{N}=\left[\overline{\mathbf{n}}_{1}, \ldots, \overline{\mathbf{n}}_{K}\right]$ with $\overline{\mathbf{n}}_{j} \sim \mathcal{C N}\left(\mathbf{0}, \sigma^{2} \mathbf{I}_{M}\right)$ independent across $j \in\{1, \ldots, K\}$. The distortion noise is defined as $\boldsymbol{\Upsilon}=\left[\boldsymbol{v}_{1}, \ldots, \boldsymbol{v}_{K}\right]$ where

$$
\boldsymbol{v}_{j}=\mathbf{D}_{\epsilon}^{\frac{1}{2}}\left(\sum_{i=1}^{K} \sqrt{\varrho_{i}} \mathbf{D}_{\left|\mathbf{h}_{i}\right|^{2}}+\sigma^{2} \mathbf{I}_{M}\right)^{\frac{1}{2}} \overline{\mathbf{r}}_{j}
$$

with $\overline{\mathbf{r}}_{j} \sim \mathcal{C N}\left(\mathbf{0}, \mathbf{I}_{M}\right)$, independent across $j \in\{1, \ldots, K\}$, denoting the randomness of the distortion noise. Performing a despreading operation on $\mathbf{Z}$ with the pilot of UE $k$ yields

$$
\mathbf{z}_{k}=\mathbf{Z} \frac{\phi_{k}^{*}}{\sqrt{K}}=\sqrt{\varrho_{k} K} \mathbf{h}_{k}+\overline{\mathbf{n}}+\sum_{j=1}^{K} \boldsymbol{v}_{j} \frac{\phi_{j k}^{*}}{\sqrt{K}}
$$

where $\overline{\mathbf{n}}=\sum_{j=1}^{K} \overline{\mathbf{n}}_{j} \frac{\phi_{j k}^{*}}{\sqrt{K}} \sim \mathcal{C N}\left(\mathbf{0}, \sigma^{2} \mathbf{I}_{M}\right)$. Then a linear minimum mean squared error (LMMSE) estimate of the channel $\mathbf{h}_{k}$ to UE $k$ can be obtained from $\mathbf{z}_{k}$. This result is summarized by the next lemma.

Lemma 1. The LMMSE estimate of $\mathbf{h}_{k}$ based on the observation $\mathbf{z}_{k}$ for $k \in\{1, \ldots, K\}$ is given by

$$
\hat{\mathbf{h}}_{k}=\frac{1}{\sqrt{\varrho_{k} K}} \mathbf{D}_{z}^{-1} \mathbf{z}_{k}
$$

where

$$
\mathbf{D}_{z}=\mathbf{I}_{M}+\frac{1}{K} \mathbf{D}_{\epsilon} \sum_{i=1}^{K} \frac{\varrho_{i} \beta_{i}}{\varrho_{k} \beta_{k}}+\frac{\sigma^{2}}{K \varrho_{k} \beta_{k}}\left(\mathbf{I}_{M}+\mathbf{D}_{\epsilon}\right) .
$$

Proof: It follows from applying LMMSE estimation techniques to the problem at hand [12, Ch. 12].

\section{Achievable SE}

To calculate an achievable SE expression, standard bounding techniques on the Massive MIMO capacity are applied. Consider a maximum ratio combining (MRC) detector defined as $\mathbf{v}_{k}=\hat{\mathbf{h}}_{k}$, then an estimate of an arbitrary data symbol in the coherence block transmitted by UE $k$ is given by

$$
\begin{aligned}
\hat{s}_{k}=\mathbf{v}_{k}^{H} \mathbf{y}= & \left(\mathbb{E}\left\{\mathbf{v}_{k}^{H} \mathbf{h}_{k}\right\}+\left(\mathbf{v}_{k}^{H} \mathbf{h}_{k}-\mathbb{E}\left\{\mathbf{v}_{k}^{H} \mathbf{h}_{k}\right\}\right)\right) \sqrt{p_{k}} s_{k} \\
& +\sum_{i \neq k}^{K} \sqrt{\rho_{i}} \mathbf{v}_{k}^{H} \mathbf{h}_{i} s_{i}+\mathbf{v}_{k}^{H} \mathbf{n}+\mathbf{v}_{k}^{H} \mathbf{e}
\end{aligned}
$$

The data estimate in (8) corresponds to an equivalent singleinput single-output (SISO) system with deterministic gain, given by $\mathbb{E}\left\{\mathbf{v}_{k}^{H} \mathbf{h}_{k}\right\}$, and non-Gaussian noise for which an achievable SE expression (i.e., lower bound on the capacity) can be obtained. This result is summarized in the next theorem.

Theorem 1. An achievable SE expression (in bit per channel use [bpcu]) for $U E k, \forall k \in\{1, \ldots, K\}$ with $M R C$ is

$$
\mathrm{R}_{k}=\left(1-\frac{K}{\tau_{c}}\right) \log _{2}\left(1+\operatorname{SINR}_{k}\right)
$$

where $\mathrm{SINR}_{k}$ is the effective signal to interference plus noise ratio (SINR) of UE k shown in (10) and (11) at the top of the next page.

Proof: It follows from deriving a lower bound on the capacity of the equivalent SISO system shown in (8) with deterministic gain $\mathbb{E}\left\{\mathbf{v}_{k}^{H} \mathbf{h}_{k}\right\}$ and non-Gaussian noise [1, Ch. 2]. The closed-form expression of $\mathrm{SINR}_{k}$ in (11) is obtained by applying known properties of circularly symmetric complex Gaussian random vectors [4, Appx. A]. 


$$
\begin{aligned}
\operatorname{SINR}_{k} & =\frac{\left|\mathbb{E}\left\{\mathbf{v}_{k}^{H} \mathbf{h}_{k}\right\}\right|^{2} \rho_{k}}{\left(\mathbb{E}\left\{\left|\mathbf{v}_{k}^{H} \mathbf{h}_{k}\right|^{2}\right\}-\left|\mathbb{E}\left\{\mathbf{v}_{k}^{H} \mathbf{h}_{k}\right\}\right|^{2}\right) \rho_{k}+\sum_{i=1, i \neq k}^{K} \mathbb{E}\left\{\left|\mathbf{v}_{k}^{H} \mathbf{h}_{i}\right|^{2}\right\} \rho_{i}+\mathbb{E}\left\{\left|\mathbf{v}_{k}^{H} \mathbf{n}\right|^{2}\right\}+\mathbb{E}\left\{\left|\mathbf{v}_{k}^{H} \mathbf{e}\right|^{2}\right\}} \\
= & \frac{\operatorname{tr}\left(\mathbf{D}_{z}^{-1}\right)^{2} \rho_{k} \beta_{k}}{\operatorname{tr}\left(\mathbf{D}_{\epsilon} \mathbf{D}_{z}^{-2}\right) \rho_{k} \beta_{k}+\frac{1}{K} \operatorname{tr}\left(\mathbf{D}_{\epsilon}\left(\mathbf{I}_{M}+\mathbf{D}_{\epsilon}\right) \mathbf{D}_{z}^{-2}\right) \sum_{i=1}^{K} \frac{\varrho_{i} \beta_{i}}{\varrho_{k} \beta_{k}} \rho_{i} \beta_{i}+\left(\sum_{i=1}^{K} \beta_{i} \rho_{i}+\sigma^{2}\right) \operatorname{tr}\left(\left(\mathbf{I}_{M}+\mathbf{D}_{\epsilon}\right) \mathbf{D}_{z}^{-1}\right)}
\end{aligned}
$$

\section{EFFeCt of Mixed ADC RESOlution}

Majorization theory is used in this section to analyze the impact of different levels of hardware impairments across the antenna array. In this paper, the main source of the hardware impairments are considered to be the ADCs. Thus, recall $\epsilon_{m}=\zeta_{m} 2^{-b_{m}}$ and for mathematical tractability let us assume $b_{m} \in \mathbb{R}$ to be a continuous variable (the ordering results also apply to integer values), then consider the following definitions.

Definition 1. Let $\bar{b}>0, \quad \mathbf{b}=\left[b_{1}, \ldots, b_{M}\right]^{T}$ with $b_{1} \geq b_{2} \geq \cdots \geq b_{M} \geq 1$ and

$$
\mathcal{L}=\left\{\mathbf{b}: \sum_{m=1}^{M}[\mathbf{b}]_{m}=\bar{b}\right\} .
$$

Then, for $\mathbf{b}_{1}, \mathbf{b}_{2} \in \mathcal{L}$

$$
\mathbf{b}_{1} \succ \mathbf{b}_{2} \text { if } \sum_{m=1}^{l}\left[\mathbf{b}_{1}\right]_{m} \geq \sum_{m=1}^{l}\left[\mathbf{b}_{2}\right]_{m}, l \in\{1, \ldots, M-1\} \text {. }
$$

When $\mathbf{b}_{1} \succ \mathbf{b}_{2}$ it is said that $\mathbf{b}_{1}$ majorizes $\mathbf{b}_{2}$ [13]. In other words, the total amount of $A D C$ bits $\bar{b}$ is spread less equally in $\mathbf{b}_{1}$ than in $\mathbf{b}_{2}$.

Definition 2. A function $f(\boldsymbol{b}): \mathbb{R}^{M} \rightarrow \mathbb{R}$ is said to be Schurconvex (Schur-concave) if for any $\mathbf{b}_{1}, \mathbf{b}_{2} \in \mathcal{L}$ it holds that

$$
\mathbf{b}_{1} \succ \mathbf{b}_{2} \Rightarrow \begin{cases}f\left(\mathbf{b}_{1}\right) \geq f\left(\mathbf{b}_{2}\right) & \text { Schur-convex } \\ f\left(\mathbf{b}_{1}\right) \leq f\left(\mathbf{b}_{2}\right) & \text { Schur-concave }\end{cases}
$$

\section{A. Impact on Achievable SE}

From (11) the SINR of UE $k$ can be rewritten as a function of $\mathbf{b} \in \mathcal{L}$ such that $\operatorname{SINR}_{k}(\mathbf{b})=\frac{\left(\sum_{m=1}^{M} f\left([\mathbf{b}]_{m}\right)\right)^{2}}{\sum_{m=1}^{M} g\left([\mathbf{b}]_{m}\right)}$ where

$$
\begin{aligned}
f(b) & =\frac{1}{1+\overline{\mathrm{B}}+(\overline{\mathrm{A}}+\overline{\mathrm{B}}) \zeta^{2} 2^{-2 b}}, \\
g(b) & =\frac{\zeta^{2} 2^{-2 b}\left(1+\mathrm{A}\left(1+\zeta^{2} 2^{-2 b}\right)\right)}{\left(1+\overline{\mathrm{B}}+(\overline{\mathrm{A}}+\overline{\mathrm{B}}) \zeta^{2} 2^{-2 b}\right)^{2}}+\frac{\mathrm{B}\left(1+\zeta^{2} 2^{-2 b}\right)}{1+\overline{\mathrm{B}}+(\overline{\mathrm{A}}+\overline{\mathrm{B}}) \zeta^{2} 2^{-2 b}}
\end{aligned}
$$

are scalar functions with

$$
\begin{aligned}
& \mathrm{A}=\frac{1}{K}\left(1+\sum_{i \neq k}^{K} \frac{\varrho_{i} \rho_{i}}{\varrho_{k} \rho_{k}}\left(\frac{\beta_{i}}{\beta_{k}}\right)^{2}\right)>0, \\
& \mathrm{~B}=1+\sum_{i \neq k}^{K} \frac{\rho_{i}}{\rho_{k}} \frac{\beta_{i}}{\beta_{k}}+\frac{\sigma^{2}}{\rho_{k} \beta_{k}}>1,
\end{aligned}
$$

$$
\overline{\mathrm{A}}=\frac{1}{K}\left(1+\sum_{i \neq k}^{K} \frac{\varrho_{i}}{\varrho_{k}} \frac{\beta_{i}}{\beta_{k}}\right)>0, \quad \overline{\mathrm{B}}=\frac{\sigma^{2}}{K \varrho_{k} \beta_{k}}>0 .
$$

In practice, the uplink power is controlled so that the received signal strength from UEs with high channel gain does not overshadow the one of UEs with low channel gain. This is also important to maintain a low dynamic range for the ADCs, especially in Massive MIMO where low-resolution ADCs are expected to be used. Thus, to gain insights into the behavior of $f(\cdot)$ and $g(\cdot)$, let us assume statistical channel inversion power control where $\rho_{k}=\frac{\rho}{\beta_{k}}$ and $\varrho_{k}=\frac{\varrho}{\beta_{k}} \forall k$, resulting in ${ }^{1}$

$$
\mathrm{A}=\overline{\mathrm{A}}=1, \quad \mathrm{~B}=K+\frac{\sigma^{2}}{\rho}, \quad \overline{\mathrm{B}}=\frac{\sigma^{2}}{K \varrho}
$$

such that

$$
\begin{aligned}
& f(b)=\frac{1}{(1+\overline{\mathrm{B}})\left(1+\zeta^{2} 2^{-2 b}\right)}, \\
& g(b)=\frac{\zeta^{2} 2^{-2 b}\left(2+\zeta^{2} 2^{-2 b}\right)}{(1+\overline{\mathrm{B}})^{2}\left(1+\zeta^{2} 2^{-2 b}\right)^{2}}+\frac{\mathrm{B}}{(1+\overline{\mathrm{B}})} .
\end{aligned}
$$

It is of interest to investigate if the SE preserves the ordering conditions in Definition 2. In other words, given a fixed budget for the total number of ADC bits available at the BS, meaning $\sum_{m=1}^{M} b_{m}=\bar{b}$, would it be more beneficial to concentrate the bits on a small number of antennas (Schur-convex) or to spread them equally across the antennas (Schur-concave)? This condition is summarized in the next lemma.

Lemma 2. Let $b_{(1)}$ and $b_{(2)}$ be first and second largest elements of $\mathbf{b} \in \mathcal{L}$ and

$$
\begin{aligned}
\Delta \operatorname{SINR}_{k}= & 2 \underbrace{\left(f^{\prime}\left(b_{(1)}\right)-f^{\prime}\left(b_{(2)}\right)\right)}_{\Delta f^{\prime}}\left(\sum_{m=1}^{M} g\left(b_{m}\right)\right) \\
& -\underbrace{\left(g^{\prime}\left(b_{(1)}\right)-g^{\prime}\left(b_{(2)}\right)\right)}_{\Delta g^{\prime}}\left(\sum_{m=1}^{M} f\left(b_{m}\right)\right)
\end{aligned}
$$

where $f^{\prime}(\cdot)$ and $g^{\prime}(\cdot)$ refer to the first derivative of $f(\cdot)$ and $g(\cdot)$, respectively. A necessary and sufficient condition for $\mathrm{SINR}_{k}(\mathbf{b})$ to be Schur-convex (or Schur-concave) is

$$
\Delta \operatorname{SINR}_{k} \begin{cases}\geq 0 & \Rightarrow \text { Schur-convex } \\ \leq 0 & \Rightarrow \text { Schur-concave. }\end{cases}
$$

Proof: It follows from the symmetry of $\operatorname{SINR}_{k}(\mathbf{b})$ (since the BS antennas are not ordered in any particular way) and

\footnotetext{
${ }^{1}$ In practice, this holds approximately for other power control methods.
} 
the simplification of Schur's condition [13, Ch. 3] considering $f(\cdot)$ and $g(\cdot)$ defined as in (19) and (20) respectively.

Notice that the sign of $\Delta \mathrm{SINR}_{k}$ depends on the sign of $\Delta f^{\prime}$ and $\Delta g^{\prime}$ in (21) which are given by the convexity of $f(\cdot)$ and $g(\cdot)$. Thus, the second order derivatives of $f(\cdot)$ and $g(\cdot)$ defined in (19) and (20), respectively, are given by

$$
\begin{aligned}
f^{\prime \prime}(b) & =-\frac{\zeta^{2} 2^{2(b+1)}\left(2^{2 b}-\zeta^{2}\right)}{\log _{2}^{2}(e)(\overline{\mathrm{B}}+1)\left(2^{2 b}+\zeta^{2}\right)^{3}}, \\
g^{\prime \prime}(b) & =\frac{2^{4(b+1)} \zeta^{2}\left(2^{2 b-1}-\zeta^{2}\right)}{\log _{2}^{2}(e)(\overline{\mathrm{B}}+1)^{2}\left(2^{2 b}+\zeta^{2}\right)^{4}},
\end{aligned}
$$

where $e$ is the base of the natural logarithm. Note that $f^{\prime \prime}(b)<0$ for $b \geq \log _{2}(\zeta)$ which always holds when $b \geq 1$ and means that $\Delta f^{\prime}<0$ (since $f^{\prime}(b)$ is a decreasing function). Moreover, $g^{\prime \prime}(b)>0$ for $b \geq \log _{2}(\zeta)+0.5$ and always holds when $b \geq 1.5$, which means that $\Delta g^{\prime}>0$ (since $g^{\prime}(b)$ is an increasing function). Thus, for $b \geq \log _{2}(\zeta)+0.5, f(b)$ and $g(b)$ are concave and convex functions, respectively, meaning $\Delta \mathrm{SINR}_{k}<0$ (see (21)). This indicates that the SINR is a Schur-concave function and therefore the SE is maximized when the ADC bits are spread equally across the antenna array. In the case where $b<\log _{2}(\zeta)+0.5, f^{\prime \prime}(b)<0$ and $g^{\prime \prime}(b)<0$, which means that Schur's condition depends on the relative size of the terms in (21) and numerical analysis is needed to obtain more insights, this is provided in Section VI.

\section{B. Impact on Power Consumption}

The power consumption at the BS is modeled as [2], [5]

$$
\mathrm{P}=\mathrm{P}_{\mathrm{TX}}+\mathrm{C}_{0}+\mathrm{D}_{0} M+\mathrm{P}_{\mathrm{CE}}+\mathrm{P}_{\mathrm{LP}}+2 \sum_{m=1}^{M} \mathrm{P}_{\mathrm{ADC} m}
$$

where

$$
\begin{aligned}
& \mathrm{P}_{\mathrm{TX}}=\frac{B_{\mathrm{W}}}{\eta} \sum_{i=1}^{K}\left(\frac{K}{\tau_{c}} \varrho_{i}+\left(1-\frac{K}{\tau_{c}}\right) \rho_{i}\right) \\
& \mathrm{P}_{\mathrm{CE}}+\mathrm{P}_{\mathrm{LP}}=\frac{2 K B_{\mathrm{W}}}{L} \sum_{m=1}^{M} b_{m}, \quad \mathrm{P}_{\mathrm{ADC} m}=\mathrm{D}_{1} 2^{\varphi_{m} b_{m}} .
\end{aligned}
$$

The efficiency of every power amplifier is denoted by $\eta>0$, $\mathrm{C}_{0}>0$ accounts for constant power consumption (e.g., site cooling), and $D_{0}>0$ is the power consumption per $\mathrm{BS}$ transceiver chain that does not depend on $\epsilon_{m} ; \mathrm{P}_{\mathrm{CE}}$ and $\mathrm{P}_{\mathrm{LP}}$ account for the channel estimation and linear processing power consumption, respectively, where $L$ is the computational efficiency of the microprocessors measured in bitoperations/Joule. The term $\mathrm{P}_{\mathrm{ADC} m}$ represents the power consumption of the ADC in the $m^{t h}$ BS antenna (defined in (27)), where $D_{1}>0$ and $\varphi_{m}>0$ are set constants related to the figure-of-merit of ADCs [5]. By considering $\mathrm{P}(\mathbf{b})$ to be a function of $\mathbf{b} \in \mathcal{L}$, the power consumption can be shown to be increasing, convex and symmetric (since the antennas are not ordered in a particular way) which in turn makes it Schurconvex with respect to the ADC resolution. This means that the power consumption is minimized when the ADC bits are spread equally across the antenna array.

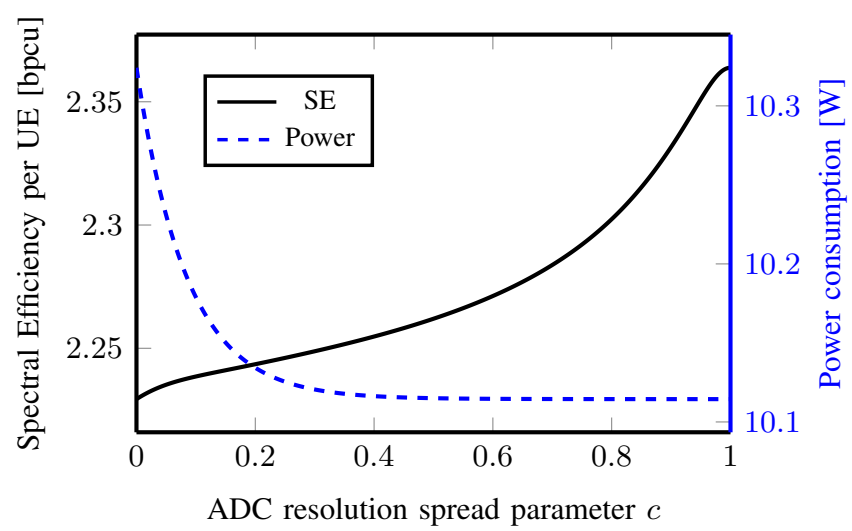

Fig. 1: SE and power consumption versus the ADC resolution spread parameter $c$ for $M=100, K=10, \zeta_{m}=1.6 \forall m$, $\rho / \sigma^{2}=1(\mathrm{SNR}=0[\mathrm{~dB}]), b^{\max }=18$ and $B_{\mathrm{W}}=20 \mathrm{MHz}$.

\section{NUMERICAL RESULTS}

Monte Carlo simulations are performed to illustrate and validate the theoretical analysis conducted in the previous sections. Consider a Massive MIMO system serving $K=10$ UEs with statistical channel inversion power control so that $\rho_{k}=\varrho_{k}=\rho / \beta_{k}$ where $\rho / \sigma^{2}$ is the average signal to noise ratio (SNR) per UE. To illustrate the results in Section $\mathrm{V}$, the parameter $c \in[0,1]$ is defined to quantify how equal the ADC bits are allocated across the antenna array. The extreme cases indicate: i) $c=0$, one antenna has $\mathrm{ADC}$ resolution $b^{\max }>1$ while all the others have one bit; ii) $c=1$, all antennas have the same ADC resolution. The ADC resolution of the $m^{\text {th }}$ antenna element is then selected as

$$
\left[\mathbf{b}_{c}\right]_{m}= \begin{cases}b^{\max } & \text { for } c=0 \text { and } m=1 \\ 1 & \text { for } c=0 \text { and } m \neq 1 \\ \frac{\left(b^{\max }-1\right)(1-c)}{1-c^{M}} c^{m-1}+1 & \text { for } 0<c<1 \\ 1+\frac{b^{\text {max }}-1}{M} & \text { for } c=1\end{cases}
$$

such that $\mathbf{b}_{c} \in \mathcal{L}$ with $1 \leq\left[\mathbf{b}_{c}\right]_{m} \leq b^{\max }$ and $\sum_{m=1}^{M}\left[\mathbf{b}_{c}\right]_{m}=M+b^{\max }-1, \forall c \in[0,1]$. This selection also implies that $\mathbf{b}_{c} \succ \mathbf{b}_{c^{\prime}}$ for $c<c^{\prime}$. Fig. 1 depicts the SE and power consumption ${ }^{2}$ as a function of $c$ where it can be seen that equal-resolution ADCs across the array jointly maximize the SE and minimize the power consumption. However, it is worth noticing that the differences are rather small.

For validation purposes, an equivalent system with exact quantization is implement such that the received signal at the BS after quantization is $\mathbf{Y}_{q}=\mathbb{Q}\left(\sum_{i=1}^{K} \mathbf{h}_{i} \mathbf{x}_{i}^{T}+\mathbf{N}\right)$. Here, $\mathbf{x}_{i}$ is the transmitted signal from UE $i$, which consists of either a pilot sequence of length $K$ defined as in Section III or data symbols given by $\sqrt{\rho_{i}} \mathbf{s}_{i} \in \mathbb{C}^{\left(\tau_{c}-K\right) \times 1} .3$ The noise matrix is $\mathbf{N}$ with elements $[\mathbf{N}]_{m j} \sim \mathcal{C N}\left(0, \sigma^{2}\right)$. The operator $\mathbb{Q}(\cdot)$ corresponds to quantizing with optimized levels as in [14] for

\footnotetext{
${ }^{2}$ The parameters for the power consumption model are found in [2], [5].

${ }^{3}$ Note that since $K$ samples are used for pilots, $\tau_{c}-K$ are left for data. For the results in Fig. 2, $\mathbf{s}_{i} \sim \mathcal{C N}\left(\mathbf{0}, \mathbf{I}_{\tau_{c}-K}\right)$.
} 


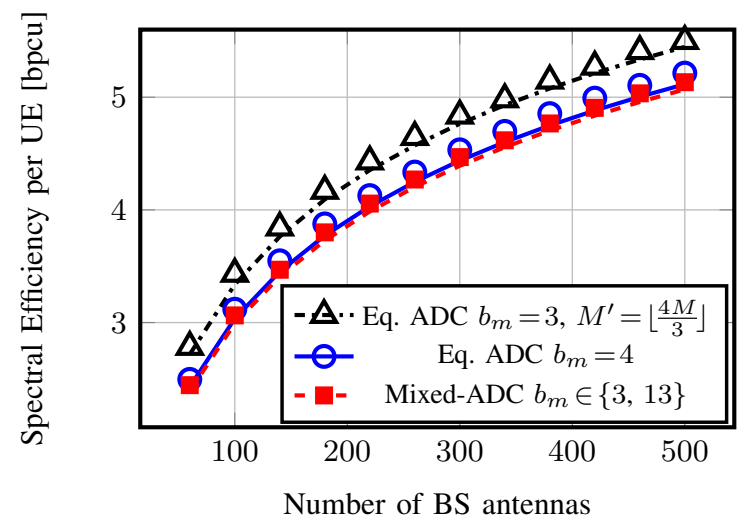

Fig. 2: Spectral efficiency per UE versus the number of antennas for $\rho / \sigma^{2}=1(\mathrm{SNR}=0[\mathrm{~dB}])$ and $K=10$. The markers correspond to (29) and the lines to (9).

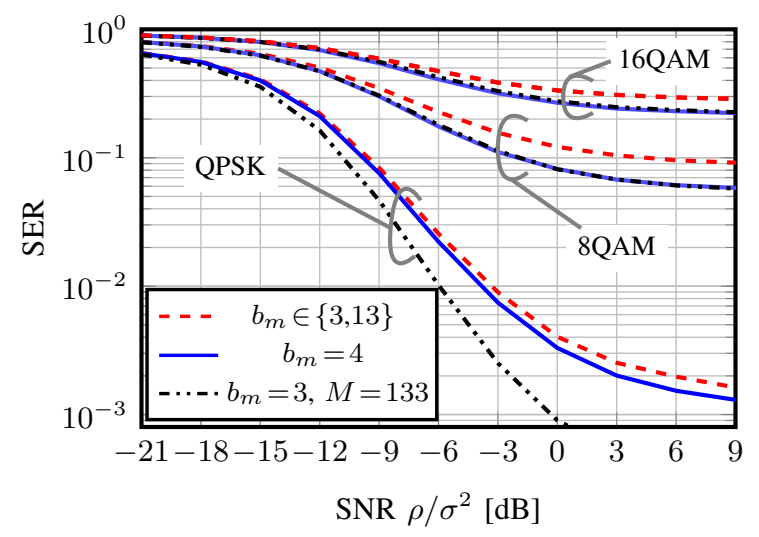

Fig. 3: SER per UE versus SNR for $M=100$ and $K=10$.

ADC resolutions of up to $b=5$ bits and for $b>5$ with uniform levels optimized as in [15]. The channel estimates for UE $k$ are computed as in Section III by the inner product of the quantized received pilot signals with $\phi_{k}$. MRC with the detector $\mathbf{v}_{k}=\hat{\mathbf{h}}_{k} /\left\|\hat{\mathbf{h}}_{k}\right\|^{2}$ is used where the normalization corresponds to an equalization operation. The data estimate for an arbitrary symbol $j$ is $\left[\hat{\mathbf{s}}_{k}\right]_{j}=\left[\mathbf{v}_{k}^{H} \mathbf{Y}_{q}\right]_{j}$, with $\mathbf{x}_{i}=\sqrt{\rho_{i}} \mathbf{s}_{i}$. An achievable SE expression for the quantized system is ${ }^{4}$

$$
\mathrm{R}_{k}=\left(1-\frac{K}{\tau_{c}}\right) \log _{2}\left(1+\frac{\left|\mathbb{E}\left\{\left[\hat{\mathbf{s}}_{k}\right]_{j}\left[\mathbf{s}_{k}\right]_{j}^{*}\right\}\right|^{2}}{\operatorname{Var}\left(\left[\hat{\mathbf{s}}_{k}\right]_{j}-\mathbb{E}\left\{\left[\hat{\mathbf{s}}_{k}\right]_{j}\left[\mathbf{s}_{k}\right]_{j}^{*}\right\}\left[\mathbf{s}_{k}\right]_{j}\right)}\right)
$$

where $\operatorname{Var}(\cdot)$ is the variance operator. In Figs. 2 and 3, three cases are shown: $i$ ) Mixed resolution ADCs with $M$ antennas where $10 \%$ of them have 13 bits and the rest 3 bits; ii) Equal-resolution ADCs of 4 bits with $M$ antennas; iii) Equalresolution ADCs of 3 bits with a larger array of $M^{\prime}=\lfloor 4 M / 3\rfloor$ antennas. Note that the sum of ADC resolutions of the whole array are almost the same for all cases. Fig. 2 depicts the SE

\footnotetext{
${ }^{4}$ This expression is computed by separating $\left[\hat{\mathbf{s}}_{k}\right]_{j}$ into two parts that are correlated and uncorrelated to $\left[\mathbf{s}_{k}\right]_{j}$, respectively, by means of the orthogonality principle, followed by known capacity bounds as in Section IV.
}

as a function of $M$ where it can be seen that equal-resolution ADCs have higher SE. Furthermore, these results confirm the validity of the parametric model introduced in Section II. Finally, Fig. 3 shows symbol error rate (SER) curves with exact quantization and QPSK, 8-QAM and 16-QAM symbols versus the SNR. The results show that having equal-resolution ADCs results in lower SER.

\section{CONCLUSION}

The design of Massive MIMO BSs calls for low-cost and low-power hardware per antenna element that in turn increases the level of distortion in the system. This work studied the per-antenna hardware design in terms of ADC resolution. An achievable SE expression was derived to account for the effect of individual per-antenna hardware impairments using a parametrized model. Majorization theory was then used to prove that having equal-resolution ADCs in the antenna array jointly maximizes the SE and minimizes the power consumption. Numerical results with exact quantization validate the theoretical analysis and system model showing that equal-resolution ADCs provide lower SER and higher SE than mixed-resolution ADCs.

\section{REFERENCES}

[1] T. L. Marzetta, E. G. Larsson, H. Yang, and H. Q. Ngo, Fundamentals of Massive MIMO. Cambridge Press, 2016.

[2] E. Björnson, L. Sanguinetti, J. Hoydis, and M. Debbah, "Optimal design of energy-efficient multi-user MIMO systems: Is massive MIMO the answer?" IEEE Trans. Wireless Commun., vol. 14, no. 6, pp. 3059-3075, Jun. 2015

[3] E. Björnson, J. Hoydis, M. Kountouris, and M. Debbah, "Massive MIMO systems with non-ideal hardware: Energy efficiency, estimation, and capacity limits," IEEE Trans. Inf. Theory, vol. 60, no. 11, pp. 71127139, Nov. 2014

[4] E. Björnson, M. Matthaiou, and M. Debbah, "Massive MIMO with nonideal arbitrary arrays: Hardware scaling laws and circuit-aware design," IEEE Trans. Wireless Commun., vol. 14, no. 8, pp. 4353-4368, Aug. 2015.

[5] D. Verenzuela, E. Björnson, and M. Matthaiou, "Hardware design and optimal ADC resolution for uplink massive MIMO systems," in Proc. IEEE SAM, Jul. 2016.

[6] C. Desset and L. V. der Perre, "Validation of low-accuracy quantization in massive MIMO and constellation EVM analysis," in Proc. EuCNC, Jun. 2015, pp. 21-25.

[7] S. Jacobsson, G. Durisi, M. Coldrey, U. Gustavsson, and C. Studer, "Throughput analysis of massive MIMO uplink with low-resolution ADCs," IEEE Trans. Wireless Commun., vol. 16, no. 6, pp. 4038-4051, Jun. 2017.

[8] C. Mollén, J. Choi, E. G. Larsson, and R. W. H. Jr., "Achievable uplink rates for massive MIMO with coarse quantization," in Proc. IEEE ICASSP, Mar. 2017.

[9] N. Liang and W. Zhang, "Mixed-ADC massive MIMO," IEEE J. Sel. Areas Commun., vol. 34, no. 4, pp. 983-997, Apr. 2016.

[10] T. C. Zhang, C. K. Wen, S. Jin, and T. Jiang, "Mixed-ADC massive MIMO detectors: Performance analysis and design optimization," IEEE Trans. Wireless Commun., vol. 15, no. 11, pp. 7738-7752, Nov. 2016.

[11] H. Pirzadeh and L. Swindlehurst, "On the optimality of mixed-ADC massive MIMO with MRC detection," in Proc. ITG WSA, Mar. 2017.

[12] S. M. Kay, Fundamentals of Statistical Signal Processing: Estimation Theory. Prentice Hall, 1993.

[13] A. W. Marshall, I. Olkin, and B. C. Arnold, Inequalities : theory of majorization and its applications. Springer New York, 2011.

[14] J. Max, "Quantizing for minimum distortion," IEEE Trans. Inf. Theory, vol. 6, no. 1, pp. 7-12, Mar. 1960.

[15] D. Hui and D. L. Neuhoff, "Asymptotic analysis of optimal fixed-rate uniform scalar quantization," IEEE Trans. Inf. Theory, vol. 47, no. 3 , pp. 957-977, Mar. 2001. 